\title{
Nonlocal boundary value problem in terms of flow for Sturm-Liouville operator in differential and difference statements
}

\author{
Dovlet M. Dovletov \\ Communicated by Makhmud Sadybekov
}

\begin{abstract}
Sturm-Liouville operator with second kind of nonlocal boundary value conditions is considered. For the classical solution, a priori estimate is established and unique existence is proved. Associated finite-difference scheme is proposed on uniform mesh and second-order accuracy for approximation is proved. An application of obtained results to nonlocal boundary problems with weight integral conditions is provided.
\end{abstract}

Keywords. Sturm-Liouville operator, nonlocal boundary value problem, second kind of nonlocal boundary conditions, a priori estimate, finite-difference scheme, second-order of accuracy approximation.

2010 Mathematics Subject Classification. 34B10, 65L10.

\section{Introduction}

For the first time, nonlocal boundary value problems (NLBVP) were considered by M. Picone [1], A. Sommerfeld [2], R. Mises [3] and J. D. Tamarkin [4] at the beginning of 20th century. Modern research on NLBVP for last fifty years was strongly motivated by two-page joint article of A. V. Bitsadze and A. A. Samarskii [5]. V. A. Il'in and E. I. Moiseev, in their joint works [6, 7], considered differential and difference statements of NLBVP for the Sturm-Liouville operator with first kind of nonlocal boundary (NLB) conditions

$$
\begin{aligned}
& \left(k(x) u^{\prime}(x)\right)^{\prime}-q(x) u(x)=-f(x), \quad x \in(0,1), \\
& u(0)=0, u(1)=\sum_{k=1}^{n} \alpha_{k} u\left(\xi_{k}\right)
\end{aligned}
$$

and with second kind of NLB conditions

$$
\begin{aligned}
& \left(k(x) u^{\prime}(x)\right)^{\prime}-q(x) u(x)=-f(x), \quad x \in(0,1), \\
& u(0)=0, \Pi(1)=\sum_{k=1}^{n} \alpha_{k} \Pi\left(\xi_{k}\right),
\end{aligned}
$$


where NLB conditions are written in terms of flow function $\Pi(x)=k(x) u^{\prime}(x)$, respectively.

First and second kinds of NLBVP, formulated in [6,7], were considered under assumption that nonlocality factors $\alpha_{k}$ have the same signs. For classical solution of differential NLBVP, a priori estimations were established and theorems on unique existence were proved. Associated finite-difference schemes for numerical approximation of above mentioned NLBVP were proposed on uniform mesh with second-order of accuracy.

V. A. I'in and E. I. Moiseev in [8] investigated criteria for a priori estimations of solutions for a wide class of conjugate nonlocal problems for the Sturm-Liouville operator with first, second, mixed and integral kinds of NLB conditions.

Subsequently to [6,7], some variations for first kind NLBVP for ordinary secondorder linear differential equations with different signs on nonlocality factors in NLB conditions were considered by the author [9]. Other variations of first and second kinds of NLBVP with associated finite-difference schemes for secondorder ordinary linear differential equations were also considered by the author [10].

Other researches on NLBVP for second-order differential equations with multipoint, integral and functional boundary conditions were conducted in [11-15] with respect to existence and uniqueness criteria, conditions for the well-posedness, difference interpretation and physical applications.

The references for NLBVP listed in article [10] and some recent works [16-18] show an unceasing interest in NLBVP for elliptic, parabolic, hyperbolic and mixed kinds of differential equations.

The present paper is stimulated by work of V. A. Il'in and E. I. Moiseev [7] and concerns the specific variations of NLBVP regarding the Sturm-Liouville operator with NLB conditions when nonlocality factors have different signs. In the current paper, we get a priori estimations and prove unique existence for classical solution of the differential problem. We propose the second-order of accuracy difference scheme on uniform mesh for approximation of smooth solution in difference metrics $C, W_{2}^{1}$ and $W_{2}^{2}$.

\section{Differential problem}

We consider the problem

$$
\begin{aligned}
& \left(k(x) u^{\prime}(x)\right)^{\prime}-q(x) u(x)=f(x), \quad x \in(0,1), \\
& u(0)=0, \Pi(1)=\sum_{k=1}^{n} \alpha_{k} \Pi\left(\zeta_{k}\right)-\sum_{l=1}^{m} \beta_{l} \Pi\left(\eta_{l}\right)
\end{aligned}
$$


for $\Pi(x)=k(x) u^{\prime}(x), \alpha_{k}>0 \forall k=1, . ., n$ and $\beta_{l}>0 \forall l=1, \ldots, m$, $0<\zeta_{1}<\zeta_{2}<\ldots<\zeta_{n}<1$ and $0<\eta_{1}<\eta_{2}<\ldots<\eta_{m}<1$, but $\zeta_{k} \neq \eta_{l}$ $\forall k$ and $l$.

Actually, NLB conditions (2) imply that the problem introduced in [7] is considered here with nonlocality factors having different signs, i.e., when $\alpha_{k}$ have positive signs and $\left(-\beta_{l}\right)$ have negative signs, respectively. Since the flow $\Pi(x)$ is a continuous function on $[0,1]$, then by mean value (MV) property $^{1}[7, \mathrm{p} .1423]$ applied to $\Pi(x)$ we reduce our original NLB conditions (2) to the following one

$$
u(0)=0, \Pi(1)=\alpha \Pi(\zeta)-\beta \Pi(\eta)
$$

with $\alpha=\sum_{k=1}^{n} \alpha_{k}, \beta=\sum_{l=1}^{m} \beta_{l}, \zeta \in\left[\zeta_{1}, \zeta_{n}\right]$ and $\eta \in\left[\eta_{1}, \eta_{m}\right]$. These notations for $\alpha$ and $\beta$ will be used throughout the paper.

As far as $\alpha>0$ and $\beta>0$, (2') itself is a particular case of NLB conditions (2). First, we prove the following theorem.

Theorem 2.1. Let $k(x) \in C^{1}[0,1], \quad q(x) \in C^{0}[0,1], \quad f(x) \in C^{0}[0,1]$, $k(x) \geq m_{0}>0, \quad q(x) \geq 0$ on $[0,1]$. If $-\infty<\sum_{k=1}^{n} \alpha_{k}-\sum_{l=1}^{m} \beta_{l}<1$ for $\zeta_{n}<\eta_{1}$ or $0<\sum_{k=1}^{n} \alpha_{k}<1 \forall \zeta_{k}$ and $\eta_{l}$, then a priori estimation

$$
\|u(x)\|_{W_{2}^{2}(0,1)} \leq C\|f(x)\|_{L_{2}(0,1)}
$$

holds for classical solution of NLBVP (1), (2).

$$
\text { If } \sum_{k=1}^{n} \alpha_{k}-\sum_{l=1}^{m} \beta_{l}=1 \text { for } \zeta_{n}<\eta_{1} \text { or } \sum_{k=1}^{n} \alpha_{k}=1 \quad \forall \zeta_{k} \text { and } \eta_{l} \text {, then } a
$$
priori estimation (3) is valid if additionally $q(x) \geq m_{1}>0$ holds.

Proof. Assume that a classical solution of NLBVP (1), (2) exists. To establish a priori estimation (3), we consider the solution of problem (1), (2) as a solution of reduced NLBVP (1), (2').

We will prove a priori estimation (3) for the classical solution of NLBVP (1), (2') and then the validity of (3) for (1), (2) will be established.

Note, if $\zeta_{n}<\eta_{1}$, then we have $\zeta<\eta$ while $-\infty<\alpha-\beta \leq 1$ in NLB conditions (2'). With regard to other possibility for $\zeta$ and $\eta$, under assumptions of this theorem it will be sufficient ${ }^{2}$ to claim that $0<\eta<\zeta<1$ while $0<\alpha \leq 1$. Therefore, in accordance with theorem assumptions it will be enough to show the

\footnotetext{
${ }^{1}$ The MV property is applicable for any arbitrary function being continuous on closed interval.

${ }^{2}$ Because, if it occurs that $\zeta \leq \eta$, then from $0<\alpha \leq 1$ it automatically follows that $-\infty<\alpha-\beta \leq 1$ and it brings us to already designated case for (2').
} 
validity of a priori estimation (3) for the classical solution of NLBVP (1), (2') in the following two cases:

(i) $-\infty<\alpha-\beta \leq 1$ for $0<\zeta<\eta<1$;

(ii) $0<\alpha \leq 1$ for $0<\eta<\zeta<1$.

First, we consider case (i). By using MV property for $\Pi(x)$ once again we can rewrite NLB condition (2') as $(1+\beta) \Pi(\xi)=\alpha \Pi(\zeta)$ for $0<\zeta<\eta \leq \xi \leq 1$ for proper point $\xi$. Therefore, the classical solution of the reduced NLBVP (1), (2') satisfies the following NLB conditions: $u(0)=0, \Pi(\xi)=\frac{\alpha}{1+\beta} \Pi(\zeta)$ while $0<\frac{\alpha}{1+\beta} \leq 1$. Then, according to [7], for the smooth solution of equation (1) with such NLB conditions a priori estimation

$$
\|u(x)\|_{W_{2}^{2}(0, \xi)} \leq C_{1}\|f(x)\|_{L_{2}(0, \xi)}
$$

is valid. Further, since $\zeta \in(0, \xi)$ and $\eta \in(0, \xi]$, by application of embedding theorem, we get

$$
\begin{gathered}
\left|u^{\prime}(\zeta)\right| \leq C_{2}\|u(x)\|_{W_{2}^{2}(0, \xi)}, \\
\left|u^{\prime}(\eta)\right| \leq C_{2}\|u(x)\|_{W_{2}^{2}(0, \xi)}
\end{gathered}
$$

Now using (4), (5) and (3') for (2') we are able to obtain the following estimation

$$
\left|u^{\prime}(1)\right| \leq \frac{\alpha+\beta}{m_{0}} M_{0} C_{2}\|u(x)\|_{W_{2}^{2}(0, \xi)} \leq C_{3}\|f(x)\|_{L_{2}(0, \xi)} \leq C_{3}\|f(x)\|_{L_{2}(0,1)}
$$

with $k(x) \leq M_{0} \quad \forall x \in[0,1]$.

Let us treat NLBVP (1), (2') as a corresponding differential problem for equation (1) with local boundary conditions

$$
u(0)=0, u^{\prime}(1)=\gamma
$$

while

$$
|\gamma| \leq C_{3}\|f(x)\|_{L_{2}(0,1)}
$$

Subject to (8) a priori estimation (3) for local boundary value problem (1), (7) is well-known. Therefore, because (3) is valid for the solution of NLBVP (1), (2'), then it is true for the classical solution of original NLBVP (1), (2) in the case (i).

Let us consider the case (ii). Here we have $0<\eta<\zeta<1$ and demand $0<\alpha \leq 1$ for NLB conditions (2').

If $\operatorname{sgn}(\Pi(\zeta) \Pi(1))=-1$, then $\Pi(\xi)=0$ for some point $\xi \in(\zeta, 1)$. Therefore, (3'), (4), (5) and (6) are true. It brings us to corresponding local conditions (7) with the bound (8). Therefore, (3) is valid. 
If $\operatorname{sgn}(\Pi(\eta) \Pi(1))=-1$, then $\Pi(\xi)=0$ for some point $\xi \in(\eta, 1)$; therefore, $u^{\prime}(\xi)=0$. For equation (1) with local boundary conditions

$$
u(0)=0, u^{\prime}(\xi)=0
$$

a priori estimation (3) is known; therefore, inequality (5) is valid. Then the solution of reduced NLBVP (1), (2') can be treated as a solution of differential equation (1) with corresponding NLB conditions

$$
u(0)=0, \Pi(1)=\alpha \Pi(\zeta)+\gamma
$$

while

$$
|\gamma| \leq C_{4}\|f(x)\|_{L_{2}(0,1)},
$$

with $C_{4}=\beta M_{0} C_{2}$ and $k(x) \leq M_{0} \quad \forall x \in[0,1]$.

Define the function $v(x)=u(x)+q \rho(x)$ with $q=\left(\alpha k(\zeta)\left(1-\zeta^{2}\right)\right)^{-1} \gamma$, $\rho(x)=x(1-x)^{2}$ so that

$$
|q|=\left|\left(\alpha k(\zeta)\left(1-\zeta^{2}\right)\right)^{-1} \gamma\right| \leq\left(\alpha m_{0}\left(1-\zeta_{n}^{2}\right)\right)^{-1}|\gamma| \leq C_{5}\|f(x)\|_{L_{2}(0,1)} .
$$

Since $u(x)$ is a solution for (1), (10), then $v(x)$ is a corresponding solution for the next NLBVP

$$
\begin{gathered}
\left(k(x) v^{\prime}(x)\right)^{\prime}-q(x) v(x)=-h(x), \quad x \in(0,1), \\
v(0)=0, \Pi_{v}(1)=\alpha \Pi_{v}(\zeta)
\end{gathered}
$$

with $\Pi_{v}(x)=k(x) v^{\prime}(x), \quad h(x)=f(x)+q L \rho(x)$. Here $L$ denotes the SturmLiouville operator.

For NLBVP (1'), (13) with $0<\alpha \leq 1$ a priori estimation

$$
\|v(x)\|_{W_{2}^{2}(0,1)} \leq C\|h(x)\|_{L_{2}(0,1)}
$$

was proved in the article [7]. Therefore, since we have (11) and (12) regarding $h(x)$, then

$$
\begin{aligned}
\|u(x)\|_{W_{2}^{2}(0,1)} & \leq\|v(x)\|_{W_{2}^{2}(0,1)}+|q|\|\rho(x)\|_{W_{2}^{2}(0,1)} \leq \\
& \leq C\|h(x)\|_{L_{2}(0,1)}+C_{5}\|f(x)\|_{L_{2}(0,1)} \leq C_{6}\|f(x)\|_{L_{2}(0,1)}
\end{aligned}
$$

with corresponding constants $C_{5}$ and $C_{6}$.

To complete the proof of theorem we consider case (ii) when $\operatorname{sgn}(\Pi(\zeta) \Pi(1))=1$ and $\operatorname{sgn}(\Pi(\eta) \Pi(1))=1$ for NLB condition (2'). Then, 
the solution of NLBVP (1), (2') satisfies equation (1) with corresponding NLB conditions

$$
u(0)=0, \Pi(1)=(\alpha-s) \Pi(\zeta)
$$

for $s=\beta \Pi(\eta) / \Pi(\zeta)$ so that $s>0$. Since $\operatorname{sgn}(\Pi(\zeta) \Pi(1))=1$, we have $\alpha-s>0$; therefore, the solution of NLBVP (1), (2') can be treated as a solution of equation (1) with NLB conditions

$$
u(0)=0, \Pi(1)=\hat{\alpha} \Pi(\zeta)
$$

while $0<\hat{\alpha}<\alpha \leq 1$. For the solution of such problem a priori estimation (3) is valid due to results of the paper [7]. The proof of Theorem 2.1 is completed.

Note 1. To prove a priori estimation (3) we demand: $-\infty<\sum_{k=1}^{n} \alpha_{k}-\sum_{l=1}^{m} \beta_{l} \leq 1$ while $\zeta_{n}<\eta_{1}$ in particular. Let us illustrate, that this requirement is essential.

Consider $k(x)=\exp (c x), q(x)=\frac{3}{4} c^{2} \exp (c x)$ for homogenous equation (1). Then, for two arbitrary points $0<\zeta<\eta<1$ and arbitrary $\beta>0$, the function $u(x)=c^{-1}(\exp (c x / 2)-\exp (-3 c x / 2))$ assigns the solution of homogenous NL$\operatorname{BVP}(1),\left(2^{\prime}\right)$ with $\alpha=(\Pi(1)+\beta \Pi(\eta)) / \Pi(\zeta)$ for $\Pi(x)=2^{-1}(\exp (3 c x / 2)+$ $+3 \exp (-c x / 2))$. Since $\Pi(x)$ is a continuous, positive and strictly increasing function on $[0,1]$, by MV property we get $\alpha=(1+\beta) \Pi(\xi) / \Pi(\zeta)$ for some $\xi \in[\eta, 1]$; therefore, $\alpha>1+\beta$ for $\forall c \neq 0$. It means that a non-trivial solution for this example exists if $\alpha-\beta>1$, i.e. a priori estimation (3) without assumptions of Theorem 2.1 is not valid in general.

Note 2. To prove a priori estimation (3) under assumption $\sum_{k=1}^{n} \alpha_{k}-\sum_{l=1}^{m} \beta_{l}=1$ while $\zeta_{n}<\eta_{1}$ we require the limitation $q(x) \geq m_{1}>0$ in particular. It is easy to show that this bound on $q(x)$ is essential, too. Actually, if $\alpha-\beta=1$, then the homogenous equation $u^{\prime \prime}(x)=0$ with NLB conditions (2) has the non-trivial solution $u(x)=c x \forall c \neq 0$. It means that without this essential bound a priori estimation (3) is not valid in general.

Note 3. By the way, for the homogenous equation $u^{\prime \prime}(x)=0$ with NLB conditions (2) the non-trivial solution $u(x)=c x$ also exists if $\alpha>1$ and $0<\beta=\alpha-1$. It means that for $\alpha>1$ the estimation (3) is not valid in general, too.

Theorem 2.2. On the assumptions of Theorem 2.1, the classical solution of $N L B V P(1),(2)$ from $C^{2}[0,1]$ exists and it is unique.

Proof. The uniqueness of the solution follows from a priori estimation which was proved in Theorem 2.1. 
Following to $[7$, p. 1425], for an arbitrary function $F(x)$ we consider the following problem

$$
\begin{aligned}
& \left(k(x) u^{\prime}\right)^{\prime}-u / k(x)=F(x), \\
& u(0)=0, \Pi(1)=\sum_{k=1}^{n} \alpha_{k} \Pi\left(\zeta_{k}\right)-\sum_{l=1}^{m} \beta_{l} \Pi\left(\eta_{l}\right) .
\end{aligned}
$$

The solution of this problem is defined by

$$
u(x)=A \sinh P(x)+\int_{0}^{x} \sinh (P(x)-P(t) F(t) d t,
$$

where

$$
\begin{gathered}
P(x)=\int_{0}^{x}[k(\tau)]^{-1} d t \\
A=\left[\cosh P(1)-\sum_{k=1}^{n} \alpha_{k} \cosh P\left(\zeta_{k}\right)+\sum_{l=1}^{m} \beta_{l} \cosh P\left(\eta_{l}\right)\right]^{-1} \\
\times\left(\sum_{k=1}^{n} \alpha_{k} \int_{0}^{\zeta_{k}} \cosh \left(P\left(\zeta_{k}\right)-P(t)\right) F(t) d t\right. \\
-\sum_{l=1}^{m} \beta_{0}^{\eta_{l}} \cosh \left(P\left(\eta_{l}\right)-P(t)\right) F(t) d t \\
\left.\quad-\int_{0}^{1} \cosh (P(1)-P(t)) F(t) d t\right) .
\end{gathered}
$$

For the expression in square brackets of (16) the following is true:

$$
\begin{aligned}
& \cosh P(1)-\sum_{k=1}^{n} \alpha_{k} \cosh P\left(\zeta_{k}\right)+\sum_{l=1}^{m} \beta_{l} \cosh P\left(\eta_{l}\right) \\
= & \cosh P(1)-\alpha \cosh P(\hat{\zeta})+\beta \cosh P(\hat{\eta})>0 .
\end{aligned}
$$

Indeed, from MV property for continuous function $P(x)$ we have some points $\hat{\zeta} \in\left[\zeta_{1}, \zeta_{n}\right]$ and $\hat{\eta} \in\left[\eta_{1}, \eta_{m}\right]$ which satisfy the equality part in (17). Further, for these points we have the following cases: 
(i) $0<\hat{\zeta}<\hat{\eta}<1, \quad-\infty<\alpha-\beta \leq 1$ when $\zeta_{n}<\eta_{1}$; and

(ii) $0<\hat{\eta}<\hat{\zeta}<1, \quad 0<\alpha \leq 1$ when otherwise.

Since the function $P(x)$, defined by (15), is continuous, positive and strictly increasing, the following inequalities are valid for case (i):

$$
\begin{gathered}
\cosh P(1)-\alpha \cosh P(\hat{\zeta})+\beta \cosh P(\hat{\eta})>\cosh P(1)-(\alpha-\beta) \cosh P(\hat{\eta}) \\
> \begin{cases}\cosh P(1)>0, & \text { if }-\infty<\alpha-\beta \leq 0, \\
(1-(\alpha-\beta)) \cosh P(1)>0, & \text { if } 0<\alpha-\beta<1, \\
\cosh P(1)-\cosh P(\hat{\eta})>0, & \text { if } \alpha-\beta=1 ;\end{cases}
\end{gathered}
$$

and the following inequalities are valid for case (ii):

$$
\begin{gathered}
\cosh P(1)-\alpha \cosh P(\hat{\zeta})+\beta \cosh P(\hat{\eta})>\cosh P(1)-\alpha \cosh P(\hat{\zeta}) \\
\quad> \begin{cases}(1-\alpha) \cosh P(1)>0, & \text { if } 0<\alpha<1, \\
\cosh P(1)-\cosh P(\hat{\zeta})>0, & \text { if } \alpha=1 .\end{cases}
\end{gathered}
$$

Then inequality (17) is true; therefore, constant $A$ defined by (16) always exists for $u(x)$ given by expression (14).

Substitution of $F(x)=(q(x)-1 / k(x)) u(x)-f(x)$ into the formula (14) makes the problem (1),(2) equivalent to corresponding Fredholm integral equation of the second kind

$$
u(x)=\int_{0}^{1} K(x, t) u(t) d t+\hat{f}(x),
$$

where $K(x, t)=K_{0}(x, t)+K_{1}(x, t)+\sum_{k=1}^{n} Z_{k}(x, t)+\sum_{1=1}^{m} H_{l}(x, t)$ with

$$
K_{0}(x, t)= \begin{cases}\sinh (P(x)-P(t))(q(t)-1 / k(t)), & \text { if } 0 \leq x \leq 1,0 \leq t \leq x, \\ 0, & \text { if } 0 \leq x \leq 1, x<t \leq 1\end{cases}
$$

$$
\begin{aligned}
& K_{1}(x, t)=[\cosh P(1)-\alpha \cosh P(\hat{\zeta})+\beta \cosh P(\hat{\eta})]^{-1} \sinh P(x) \\
& \times \cosh (P(t)-P(1))(q(t)-1 / k(t)), \quad \text { if } 0 \leq x \leq 1,0 \leq t \leq 1, \\
& Z_{k}(x, t)=\left\{\begin{array}{l}
{[\cosh P(1)-\alpha \cosh P(\hat{\zeta})+\beta \cosh P(\hat{\eta})]^{-1}} \\
\times \alpha_{k} \sinh \left(P ( x ) \operatorname { c o s h } \left(P\left(\zeta_{k}-P(t)\right)\right.\right. \\
\times(q(t)-1 / k(t)), \text { if } 0 \leq x \leq 1,0 \leq t \leq \zeta_{k}, \\
0, \text { if } 0 \leq x \leq 1, \zeta_{k}<t \leq 1
\end{array}\right.
\end{aligned}
$$


for $k=1, \ldots, n$,

$$
H_{l}(x, t)=\left\{\begin{array}{l}
{[\cosh P(1)-\alpha \cosh P(\hat{\zeta})+\beta \cosh P(\hat{\eta})]^{-1}} \\
\times \beta_{l} \sinh \left(P ( x ) \operatorname { c o s h } \left(P\left(\eta_{l}-P(t)\right)\right.\right. \\
\times(q(t)-1 / k(t)), \text { if } 0 \leq x \leq 1,0 \leq t \leq \eta_{l} \\
0, \text { if } 0 \leq x \leq 1, \eta_{l}<t \leq 1
\end{array}\right.
$$

for $l=1, \ldots, m$ and

$$
\begin{aligned}
\hat{f}=-\sinh P(x)[ & \left.\cosh P(1)-\sum_{k=1}^{n} \alpha_{k} \cosh P\left(\zeta_{k}\right)+\sum_{l=1}^{m} \beta_{l} \cosh P\left(\eta_{l}\right)\right]^{-1} \\
& \times\left(\sum_{k=1}^{n} \alpha_{k} \int_{0}^{\zeta_{k}} \cosh \left(P\left(\zeta_{k}\right)-P(t)\right) f(t) d t\right. \\
& -\sum_{k=1}^{m} \beta_{l} \int_{0}^{\eta_{l}} \cosh \left(P\left(\eta_{l}\right)-P(t)\right) f(t) d t \\
-\int_{0}^{1} \cosh (P(1) & -P(t)) f(t) d t)-\int_{0}^{x} \sinh (P(x)-P(t)) f(t) d t
\end{aligned}
$$

From defined expressions for kernels $K_{0}(x, t), K_{1}(x, t), Z_{k}(x, t)(k=1, \ldots, n)$ and $H_{l}(x, t)(l=1, \ldots, m)$ it follows that each kernel is a continuous function on the square $0 \leq x \leq 1,0 \leq t \leq 1$; therefore, $K(x, t)$ is also continuous on this square. So, the Fredholm alternative is applicable for integral equation (18) in Hilbert space $L_{2}(0,1)$.

Since $K(x, t) \in C^{0}([0,1] \times[0,1])$ and $\hat{f}(x) \in C^{0}[0,1]$, the solution of integral equation (18) from $L_{2}(0,1)$ actually belongs to $C^{0}[0,1]$. Moreover, if $u(x) \in$ $C^{0}[0,1]$, then $\int_{0}^{1} K(x, t) u(t) d t$ as a function of $x$ belongs to $C^{2}[0,1]$ as soon as $k(x) \in C^{1}[0,1]$ and $q(x) \in C^{0}[0,1]$.

Furthermore, if $f(x) \in C^{0}[0,1]$, then from (19) it follows that $\hat{f}(x) \in C^{2}[0,1]$ too.

Therefore, any solution of integral equation (18) from $L_{2}(0,1)$ actually belongs to $C^{2}[0,1]$.

Now, it is enough to show that integral equation (18) has only trivial solution if $\hat{f}(x) \equiv 0$ on $[0,1]$. 
Suppose that $\hat{f}(x) \equiv 0$ on $[0,1]$ for integral equation (18). On this assumption we will show that $f(x) \equiv 0$ on $[0,1]$.

From (19) it follows that

$$
\begin{gathered}
\hat{f}^{\prime}=-\frac{\cosh P(x)}{k(x)}\left[\cosh P(1)-\sum_{k=1}^{n} \alpha_{k} \cosh P\left(\zeta_{k}\right)+\sum_{l=1}^{m} \beta_{l} \cosh P\left(\eta_{l}\right)\right]^{-1} \\
\times\left(\sum_{k=1}^{n} \alpha_{k} \int_{0}^{\zeta_{k}} \cosh \left(P\left(\zeta_{k}\right)-P(t)\right) f(t) d t-\sum_{k=1}^{m} \beta_{l} \int_{0}^{\eta_{l}} \cosh \left(P\left(\eta_{l}\right)-P(t)\right) f(t) d t\right. \\
\left.-\int_{0}^{1} \cosh (P(1)-P(t)) f(t) d t\right)-\frac{1}{k(x)} \int_{0}^{x} \cosh (P(x)-P(t)) f(t) d t .
\end{gathered}
$$

If $\hat{f}(x) \equiv 0$ on $[0,1]$, then $\hat{f}^{\prime}(x) \equiv 0$ on $[0,1]$; therefore $\hat{f}^{\prime}(0)=0$ in particular. Using last equality in (20), we have

$$
\hat{f}^{\prime}(x)=-\frac{1}{k(x)} \int_{0}^{x} \cosh (P(x)-P(t)) f(t) d t .
$$

Therefore, if $\hat{f}^{\prime}(x) \equiv 0$ on $[0,1]$, then on $[0,1]$ we have that

$$
\int_{0}^{x} \cosh (P(x)-P(t)) f(t) d t \equiv 0 .
$$

By differentiating both sides of (21) we get the following equality

$$
f(x) k(x)+\int_{0}^{x} \sinh (P(x)-P(t)) f(t) d t=0
$$

for $x \in[0,1]$. Then, by differentiating once again and taking into account (21), we get $(f(x) k(x))^{\prime}=0$ on $[0,1]$. Therefore, $f(x)=C / k(x)$ and $C$ is an arbitrary constant. Substituting this formula for $f(x)$ in (21) and integrating it, we get $f(x) \equiv 0$ on $[0,1]$ (see [7, p. 1427]). Since we have equivalency for integral equation (18) and NLBVP (1),(2), taking into account a priori estimation (3), which was proved in Theorem 2.1, we conclude that if $\hat{f}(x) \equiv 0$ on $[0,1]$, then integral equation (18) has only trivial solution. Theorem 2.2 is proved. 
Note 4. To propose a finite-difference scheme for NLBVP (1),(2) we will need much more smoothness for the solution than a twice differentiability. Therefore, we further assume that $k(x) \in C^{3}[0,1], q(x) \in C^{2}[0,1], f(x) \in C^{2}[0,1]$ and as far as $k(x) \geq m_{0}>0$ on [0,1], for the classical solution of NLBVP (1),(2) we have $u(x) \in C^{4}[0,1]$ (see [7, p. 1427]).

\section{Finite-difference scheme}

We consider a numerical statement for NLBVP (1),(2) on uniform mesh with step size $h>0, \omega_{i}=\left\{x_{i}=i h, i=1,2, \ldots, N-1 ; x_{0}=0, x_{N}=1\right\}$. For differential operator (1), we use the following finite-difference approximation (see $[19$, p.148])

$$
\Lambda y_{i}=\frac{1}{h}\left(a_{i+1} \frac{y_{i+1}-y_{i}}{h}-a_{i} \frac{y_{i}-y_{i-1}}{h}\right)-d_{i} y_{i}=-\varphi_{i}
$$

with $a_{i}=k_{i-1 / 2}=k\left(x_{i}-h / 2\right), d_{i}=q_{i}=q\left(x_{i}\right), \varphi_{i}=f\left(x_{i}\right)$. Furthermore, here we choose the step size $h$ strictly less than a half of lowest distance between any two points from the set

$$
\left\{0 \cup \zeta_{k}, k=1, \ldots, n, \cup \eta_{l}, l=1, \ldots, m, \cup 1\right\} .
$$

For NLB conditions (2), we apply the following difference approximation based on the approach from [7]

$$
\begin{aligned}
& y_{0}=0, \\
& \begin{aligned}
\mathcal{L}(h) y_{i} & =\sum_{k=1}^{n} \alpha_{k}\left\{p\left(x_{i_{k}}\right) \frac{\left[\left(i_{k}+1\right) h-\zeta_{k}\right]}{h}+p\left(x_{i_{k}+1}\right) \frac{\left[\zeta_{k}-i_{k} h\right]}{h}\right\} \\
& -\sum_{l=1}^{m} \beta_{l}\left\{p\left(x_{i_{l}}\right) \frac{\left[\left(i_{l}+1\right) h-\eta_{l}\right]}{h}+p\left(x_{i_{l}+1}\right) \frac{\left[\eta_{l}-i_{l} h\right]}{h}\right\}-p_{N}=0
\end{aligned}
\end{aligned}
$$

with $p\left(x_{i}\right)=p_{i}=2^{-1}\left[\left(a y_{\bar{x}}\right)_{i}+\left(a y_{\bar{x}}\right)_{i+1}\right]$ for $i=1,2, \ldots, N-1$, $p_{N}=\left(a y_{\bar{x}}\right)_{N}+2^{-1} d_{N} y_{N} h-2^{-1} h \varphi_{N}$. Here, numbers $i_{k}(k=1, \ldots, n)$ are defined such that $i_{k} h<\zeta_{k} \leq\left(i_{k}+1\right) h$ for each $\zeta_{k}$ and numbers $i_{l}(l=1, \ldots, m)$ are defined correspondingly such that $i_{l} h<\eta_{l} \leq\left(i_{l}+1\right) h$ for each $\eta_{l}$.

Theorem 3.1. If the step size $h$ of uniform mesh $\omega_{h}$ is small enough, then a numerical solution $y\left(x_{i}\right)$ of difference scheme (22),(23) exists, is unique and approximates the solution $u(x)$ of differential problem (1),(2) with the second-order accuracy in difference metrics $C, W_{2}^{1}$ and $W_{2}^{2}$. 
Proof. Let $z_{i}=y_{i}-u_{i}$, then $z_{i}$ is the numerical solution for the following difference scheme

$$
\begin{gathered}
\Lambda z_{i}=-\psi_{i}, \\
z_{0}=0, \mathcal{L}_{0}(h) z_{i}=-\mathcal{L}(h) u_{i}
\end{gathered}
$$

with $\mathcal{L}_{0}$ denoting $\mathcal{L}$ in $(23)$ when $\varphi_{N}=0$.

The estimation $\psi=O\left(h^{2}\right)$ holds [19, p. 151] for $\psi_{i}=\varphi_{i}+\Lambda u_{i}$. Additionally, the estimation

$$
\mathcal{L}_{0}(h) z_{i}=-\mathcal{L}(h) u_{i}=O\left(h^{2}\right)
$$

holds [7, p. 1428-1429].

Let us denote

$$
z_{i}=\tilde{z}_{i}+v_{i}
$$

with $v_{i}$ being the solution of the difference scheme

$$
\begin{gathered}
\Lambda v_{i}=\psi_{i} \\
v_{0}=0,\left(a v_{\bar{x}}\right)_{N}+2^{-1} d_{N} v_{N} h=0
\end{gathered}
$$

approximating the associated differential problem

$$
\left(k(x) v^{\prime}\right)^{\prime}-q(x) v=\psi, 0<x<1, v(0)=0, \Pi(1)=k(1) v^{\prime}(1)=0 .
$$

Since for the solution of difference problem (28),(29) the estimation

$$
\mathcal{L}_{0}(h) v_{i}=O\left(h^{2}\right)
$$

holds [7, p. 1429], $\tilde{z}$ can be considered as a solution of the following scheme

$$
\begin{gathered}
\Lambda \tilde{z}_{i}=0, \\
\tilde{z}_{0}=0, \mathcal{L}_{0}(h) \tilde{z}_{i}=O\left(h^{2}\right) .
\end{gathered}
$$

Since the numerical solution of the difference equation $\Lambda \tilde{z}_{i}=0$ conforms to A.A. Samarskii maximum principle [19, p.39-40], all $\tilde{z}_{i}$ values are non-negative or alternatively non-positive as far as $\tilde{z}_{0}=0$.

For definiteness let us consider the case when all $\tilde{z}_{i}, i=1,2, \ldots, N-1$ values are non-negative (otherwise apply $-\tilde{z}_{i}$ ). Then, from homogenous difference equation (30) it follows that

$$
0 \leq\left(a \tilde{z}_{\bar{x}}\right)_{1} \leq\left(a \tilde{z}_{\bar{x}}\right)_{2} \leq \ldots \leq\left(a \tilde{z}_{\bar{x}}\right)_{N} .
$$


Therefore, $p_{i}(\tilde{z})=2^{-1}\left[\left(a \tilde{z}_{\bar{x}}\right)_{i}+\left(a \tilde{z}_{\bar{x}}\right)_{i+1}\right], i=1, \ldots, N-1$ is a non-decreasing and non-negative mesh function. Then,

$$
0 \leq p_{i_{k}} \leq p_{i_{k}+1} \leq\left(a \tilde{z}_{\bar{x}}\right)_{N}=p_{N}^{0}-d_{N} \tilde{z}_{N} h 2^{-1} \leq p_{N}^{0}
$$

for any $\zeta_{k}, k=1, \ldots, n$ and

$$
0 \leq p_{j_{l}} \leq p_{j_{l}+1} \leq\left(a \tilde{z}_{\bar{x}}\right)_{N}=p_{N}^{0}-d_{N} \tilde{z}_{N} h 2^{-1} \leq p_{N}^{0}
$$

for any $\eta_{l}, l=1, \ldots, m$.

Now, by linear interpolation of values of mesh functions $p_{i}(\tilde{z}), i=1, \ldots, N-1$, with add-in value $p_{N}(\tilde{z})=p_{N}^{0}$ and arbitrary $p_{0}(\tilde{z})$, for example equal to zero, we define a piecewise function $\bar{p}(x)$ continuous on the whole interval $[0,1]$. By using MV property for the continuous function $\bar{p}(x)$ regarding the approximation (31), we get the following equality

$$
p_{N}^{0}(\tilde{z})=\alpha \bar{p}(\check{\zeta})-\beta \bar{p}(\check{\eta})+O\left(h^{2}\right)
$$

with $\alpha=\sum_{k=1}^{n} \alpha_{k}, \beta=\sum_{l=1}^{m} \beta_{l}$ and corresponding points $\check{\zeta} \in\left[\zeta_{1}, \zeta_{n}\right], \check{\eta} \in\left[\eta_{1}, \eta_{m}\right]$.

Since the interpolation function $\bar{p}(x)$ is non-decreasing and non-negative, the following inequalities follow from (32) directly:

$$
\begin{array}{ll}
\text { (i) } p_{N}^{0}(\tilde{z}) \leq(\alpha-\beta) \bar{p}(\check{\eta})+O\left(h^{2}\right) & \text { if } \check{\zeta} \leq \check{\eta} \text {; } \\
\text { (ii) } p_{N}^{0}(\tilde{z}) \leq \alpha \bar{p}(\check{\zeta})+O\left(h^{2}\right) & \text { if } \check{\zeta}>\check{\eta} .
\end{array}
$$

Moreover, we have

$$
\begin{array}{ll}
\text { (i) } p_{N}^{0}(\tilde{z}) \leq(\alpha-\beta) p_{N}^{0}(\tilde{z})+O\left(h^{2}\right) & \text { if } \check{\zeta} \leq \check{\eta} \text {; } \\
\text { (ii) } p_{N}^{0}(\tilde{z}) \leq \alpha p_{N}^{0}(\tilde{z})+O\left(h^{2}\right) & \text { if } \check{\zeta}>\check{\eta} .
\end{array}
$$

Therefore, the estimation

$$
p_{N}^{0}(\tilde{z})=O\left(h^{2}\right)
$$

is valid in case (i) when $-\infty<\alpha-\beta<1$ with $\check{\zeta} \leq \check{\eta}$, as well as in case (ii) when $0<\alpha<1$ with $\check{\zeta}>\check{\eta}$. It means that the numerical solution of (30),(31) can be considered as a corresponding solution of the following difference scheme:

$$
\begin{gathered}
\Lambda \tilde{z}_{i}=0, \\
\tilde{z}_{0}=0, p_{N}^{0}(\tilde{z})=O\left(h^{2}\right) .
\end{gathered}
$$


Let us show that the estimation $p_{N}^{0}(\tilde{z})=O\left(h^{2}\right)$ holds in both cases, when $\alpha-\beta=1$ for $\check{\zeta} \leq \check{\eta}$ or alternatively when $\alpha=1$ for $\check{\zeta}>\check{\eta}$.

Since we require $q(x) \geq m_{1}>0$ for above mentioned cases (i) and (ii), from difference equation (33) we have the following inequalities for mesh function $Q_{i}=\left(a \tilde{z}_{\bar{x}}\right)_{i}, i=1, \ldots, N-1$ (see [7, p. 1430-1431])

$$
\begin{gathered}
\tilde{z}_{i} / Q_{i} \geq\left(M_{0} / \xi+\xi+M_{1}\right)^{-1}=\delta>0, \\
\left.Q_{i} / Q_{N} \leq(1+(1-\xi-2 h)) m_{1} \tilde{z}_{i} / Q_{i}\right)^{-1}
\end{gathered}
$$

and therefore,

$$
Q_{i} / Q_{N} \leq(1-\bar{\delta})
$$

for some point $\xi=\max \left\{\zeta_{n}, \eta_{m}\right\}$ and corresponding constants such that $M_{0} \geq$ $k(x), M_{1} \geq q(x) \forall x \in[0,1]$.

Since $\xi$ is defined such that $i_{\xi} h<\xi \leq\left(i_{\xi}+1\right) h$, by using (35) we get valid estimations for values of th interpolation function $\bar{p}(x)$ at above mentioned points $\check{\zeta}$ and $\check{\eta}$

$$
\begin{aligned}
& \bar{p}(\check{\zeta}) \leq p_{k_{n}}(\tilde{z}) \leq p_{i_{\xi}}(\tilde{z}), \\
& \bar{p}(\check{\eta}) \leq p_{l_{m}}(\tilde{z}) \leq p_{i_{\xi}}(\tilde{z}) .
\end{aligned}
$$

Using these inequalities in (32) and taking into account (35), we obtain

$$
\begin{aligned}
p_{N}^{0}(\tilde{z}) \leq & \bar{p}_{i_{\xi}}(\check{\zeta})+O\left(h^{2}\right) \leq Q_{i_{\xi}+2}+O\left(h^{2}\right) \\
& \leq(1-\bar{\delta}) Q_{N}+O\left(h^{2}\right) \leq(1-\bar{\delta}) p_{N}^{0}(\tilde{z})+O\left(h^{2}\right)
\end{aligned}
$$

which is true for case (i) when $\alpha-\beta=1$ and case (ii) when $\alpha=1$. Therefore, the estimation $p_{N}^{0}(\tilde{z})=O\left(h^{2}\right)$ is always true for difference problem (30),(31) and hence the numerical solution of (31),(32) always can be considered as corresponding solution of (33),(34).

Now, since we have proved the estimation $p_{N}^{0}(\tilde{z})=O\left(h^{2}\right)$ for the solution of (33),(34) and because the mesh function $Q_{i}$ is non-decreasing, we have

$$
\max _{1 \leq i \leq N}\left(a \tilde{z}_{\bar{x}}\right)_{i} \leq p_{N}^{0}(\tilde{z})
$$

and therefore,

$$
\left\|\tilde{z}_{\bar{x}}\right\|=O\left(h^{2}\right) .
$$

Further, by using difference analogue of embedding theorem [19, p. 290], we have that estimation

$$
\max _{1 \leq i \leq N}\left|\tilde{z}_{i}\right|=O\left(h^{2}\right)
$$


is valid; therefore,

$$
\left\|\tilde{z}_{\bar{x} x}\right\|=O\left(h^{2}\right)
$$

holds for solution of difference equation (33).

Since we have all necessary estimations for $\tilde{z}_{i}$ and the following estimations for corresponding solution $v_{i}$ of difference problem (28),(29) (see [7, p. 1429])

$$
\left\|v_{\bar{x}}\right\|=O\left(h^{2}\right), \quad \max _{1 \leq i \leq N}\left|v_{i}\right|=O\left(h^{2}\right), \quad\left\|v_{\bar{x} x}\right\|=O\left(h^{2}\right),
$$

by using a triangle inequality in (27) we obtain the second-order accuracy for the solution of finite-difference scheme (22),(23). Theorem 3.1 is proved.

\section{Nonlocal weight integral condition}

The approach proposed by V. A. Il'in and E. I. Moiseev in [6] is also applicable to the Sturm-Liouville operator with first kind nonlocal weight integral boundary conditions

$$
\begin{aligned}
& \left(\left(k(x) u^{\prime}(x)\right)\right)^{\prime}-q(x) u(x)=-f(x), \quad x \in(0,1), \\
& u(0)=0, \quad u(1)=\int_{\zeta}^{\eta} \alpha(x) u(x) d x
\end{aligned}
$$

for function $\alpha(x)$ summable on $(\zeta, \eta)$ and $\zeta \in[0,1], \eta \in[0,1]$ in general. Actually, if $\alpha(x)$ has the same sign almost everywhere on the defined interval, then for the nonlocal differential problem mentioned above, corresponding results of [6] are applicable.

Indeed, if $\alpha(x)$ is not changing the sign and $-\infty<\int_{\zeta}^{\eta} \alpha(x) d x<1$, then by using the first mean value theorem for definite integral in above-mentioned first kind nonlocal integral condition we obtain a priori estimation (3) and unique existence of solution of above-mentioned problem as solution of two-point NLBVP ${ }^{3}$ [6].

Similarly, the approach described in [7] is applicable to second kind integral NLBVP too, i.e. for the problem

$$
\begin{aligned}
& \left(\left(k(x) u^{\prime}(x)\right)\right)^{\prime}-q(x) u(x)=-f(x), \quad x \in(0,1), \\
& u(0)=0, \quad \Pi(1)=\int_{\zeta}^{\eta} \alpha(x) \Pi(x) d x
\end{aligned}
$$

$$
{ }^{3} u(0)=0, u(1)=\alpha u(\xi), \alpha=\int_{\zeta}^{\eta} \alpha(x) d x, \xi \in(\zeta, \eta) .
$$


with above mentioned requirements on weight function $\alpha(x)$. Actually, if $\alpha(x)$ is not changing the sign and $-\infty<\int_{\zeta}^{\eta} \alpha(x) d x<1$, then by using the first mean value theorem to flow weighting integral condition, we obtain a priori estimation (3) and the unique existence of the solution of the second kind integral NLBVP as corresponding solution of two-point nonlocal problem ${ }^{4}$ which was studied in [7].

For the first and second kind integral NLB problems given above the applications of results of [6] and [7] are readily available for weight function $\int_{\zeta}^{\eta} \alpha(x) d x=$ 1 as well, but when $\zeta, \eta \in[0,1) .^{5}$ In this case, a priori estimations (3), as well as the unique existence follows from [6] and [7] by reducing integral conditions to followings conditions: $u(0)=0, u(1)=u(\xi)$ and $u(0)=0, \Pi(1)=\Pi(\xi)$ with proper $\xi$ for each problem.

Further, if some sign-changing summable weight function has finite number of sign changes in the first kind integral NLB condition, then NLBVP can be written as follows

$$
\begin{aligned}
& \left(\left(k(x) u^{\prime}(x)\right)\right)^{\prime}-q(x) u(x)=-f(x), \quad x \in(0,1), \\
& u(0)=0, \quad u(1)=\int_{\zeta}^{\hat{\zeta}} \alpha(x) u(x) d x-\int_{\breve{\eta}}^{\hat{\eta}} \beta(x) u(x) d x
\end{aligned}
$$

for corresponding summable functions $\alpha(x) \geq 0$ and $\beta(x) \geq 0, \check{\zeta} \in[0,1], \hat{\zeta} \in$ $[0,1], \check{\zeta}<\hat{\zeta}, \check{\eta} \in[0,1], \hat{\eta} \in[0,1], \check{\eta}<\hat{\eta}$. Applying the first mean value theorem for the above first kind integral condition, we get a priori estimation (3) and the unique existence of the solution of the problem given above as for solution of three-point ${ }^{6}$ NLBVP (we refer to results of [9]).

For example, if $0<\int_{\breve{\zeta}}^{\hat{\zeta}} \alpha(x) d x<1$ and the positions of points $\check{\zeta}, \hat{\zeta}, \check{\eta}, \hat{\eta}$ is arbitrary, then a priori estimation (3) and the unique existence of classical solution are the consequences of Theorems 1 and 2 from the paper [9, p. 1298-1302].

Another example, if $\hat{\zeta}<\check{\eta}$ and $-\infty<\int_{\breve{\zeta}}^{\hat{\zeta}} \alpha(x) d x-\int_{\check{\eta}}^{\hat{\eta}} \beta(x) d x<1$, then

${ }^{4} u(0)=0, \Pi(1)=\alpha \Pi(\xi), \alpha=\int_{\zeta}^{\eta} \alpha(x) d x, \xi \in(\zeta, \eta)$.

5 The reason to separate integral limits from boundary point 1 follows from proof of a priori estimation in [6] and [7].

${ }^{6} u(0)=0, u(1)=\alpha u(\zeta)-\beta u(\eta), \alpha=\int_{\breve{\zeta}}^{\hat{\zeta}} \alpha(x) d x, \beta=\int_{\check{\eta}}^{\hat{\eta}} \beta(x) d x, \zeta \in(\check{\zeta}, \hat{\zeta}), \eta \in(\check{\eta}, \hat{\eta})$. 
a priori estimation (3) and the unique existence of the classical solution follows from Theorems 1 and 2 in [9, p. 1298-1302], respectively.

Under the same restrictions on weight functions we can get (using MV property, respectively) a priori estimation and prove unique existence of classical solution for second kind integral NLBVP

$$
\begin{aligned}
& \left(\left(k(x) u^{\prime}(x)\right)\right)^{\prime}-q(x) u(x)=-f(x), \quad x \in(0,1), \\
& u(0)=0, \quad \Pi(1)=\int_{\zeta}^{\hat{\zeta}} \alpha(x) \Pi(x) d x-\int_{\breve{\eta}}^{\hat{\eta}} \beta(x) \Pi(x) d x
\end{aligned}
$$

with reference to Theorems 2.1 and 2.2. Reduced NLB conditions here are as follows: $u(0)=0, \Pi(1)=\alpha \Pi(\zeta)-\beta \Pi(\eta)$, where $\alpha=\int_{\zeta}^{\hat{\zeta}} \alpha(x) d x$ and $\beta=$ $\int_{\check{\eta}}^{\hat{\eta}} \beta(x) d x$, with proper $\zeta \in(\check{\zeta}, \hat{\zeta}), \eta \in(\check{\eta}, \hat{\eta})$.

Note that for difference interpretation of all above mentioned integral variations of NLBVP we have to require much more smoothness for weight functions to obtain the second-order accuracy for approximation. For example, requirements $\alpha(x) \in C^{2}[0,1]$ and $\beta(x) \in C^{2}[0,1]$ provide the second-order accuracy when the trapezoidal method is applied for weighting integral NLB condition.

Finally, the associated finite-difference statements will be based on results in $[6,7]$ if we have the weight function with no sign changes in the integral NLB condition. Otherwise, when we have sign-changing weighting function with finite number of sign changes, we can research finite-difference scheme by applying previous results in $[9,10]$ for the first kind NLBVP and Theorem 3.1 for the second kind NLBVP, respectively.

\section{Conclusion}

In conclusion, the author expresses his deepest deference to deceased RAS Academy Sciences academician, Prof. V. A. Il'in, whose outstanding scientific articles stimulate the author for further research.

Author expresses gratitude to RAS academician, Prof. E. I. Moiseev, whose remarkable scientific works influence the author's investigations in this area.

\section{Bibliography}

[1] M. Picone, Sui valori eccezionali di un parametro da cui dipende un'equazione differenziale lineare del secondo ordine, Ann. Scuola Norm. Sup. Pisa 11 (1910), 1-141 (In Italian). 
[2] A. Sommerfeld, Ein Beitrag zur hydrodynamische Erklärung der turbulenten Flüssigkeitsbewegungen, Proceedings of the 4th International Congress of Mathematicians, Vol. III, 116-124 (In German).

[3] R. von Mises, Beitrag zum Oszillationsproblem, in Festschrift H. Weber, Teubner, Leipzig, 1912, 252-282 (In German).

[4] J. D. Tamarkin, I. On some general problems in the theory of ordinary linear differential equations and on the expansion in series of arbitrary functions, Petrograd, 1917 (In Russian).

[5] A. V. Bisadze and A. A. Samarskii, On some simple generalizations of linear elliptic boundary problems, USSR Academy of Science Reports 185(4) (1969), 739-740.

[6] V. A. Il'in and E. I. Moiseev, First kind nonlocal boundary value problem for SturmLiouville operator in differential and difference treatment, Differential Equations 23(7) (1987), 1198-1207.

[7] V. A. Il'in and E. I. Moiseev, Second kind nonlocal boundary value problem for Sturm-Liouville operator, Differential Equations 23(8) (1987), 1422-1431.

[8] V. A. Il'in and E. I. Moiseev, An a priori estimate for the solution of a problem associated with a nonlocal boundary value problem of the first kind, Differential Equations 24(5) (1988), 519-526.

[9] D. M. Dovletov, A nonlocal boundary value problem of the first kind in a differential and a difference treatment, Differential Equations 25(8) (1989), 917-924.

[10] D. M. Dovletov, On some nonlocal boundary value problem indifferential and difference interpretation, Dissertation thesis, 128 pp., Moscow, 1989.

[11] A. M. Dovletov and D. M. Dovletov, A nonlocal boundary value problem of "third kind" for the Sturm- Liouville operator, Izv. Akad. Nauk Turkm. SSR, Ser. Fiz.Tekh., Khim. Geol. Nauk 5 (1991), 3-6 (1991; Zbl 0745.34019) (Russian. English summary).

[12] A. Lomtatidze, On a nonlocal boundary value problem for second order linear ordinary differential equations, Journal of Mathematical Analysis and Applications 193(3) (1995), 889-908.

[13] D. M. Dovletov, A nonlocal boundary value problem of second for the SturmLiouville operator, Izv. Akad. Nauk Turkm. SSR, Ser. Gumanitarn. Nauk 2 (1996), 97-97.

[14] A. Boucherif, Differential equations with nonlocal boundary conditions, Nonlinear Analysis: Theory, Methods and Applications 47(4) (2001), 2419-2430.

[15] I. T. Kiguradze and T. I. Kiguradze, Conditions for the well-posedness of nonlocal problems for second-order linear differential equations, Differential equations 47(10) (2011), 1414-1425.

[16] A. I. Kozhanov, A time-nonlocal boundary value problem for linear parabolic equations, Sib. Zh. Ind. Mat. 7(1) (2004), 51-60. 
[17] A. Ashyralyev and Y. Ozdemir, On nonolocal boundray value problems for hyperbolic-parabolic equations, Taiwanese J. Math. 11(4) (2007), 1075-1089.

[18] C. Ashyralyyev, G. Akyuz and M. Dedeturk, Approximate solution for an inverse problem of multidimensional elliptic equation with multipoint nonlocal and Neumann boundary conditions, Electron. J. Differential Equations 2017(197) (2017), $1-16$.

[19] A. A. Samarskii, Theory of Difference Schemes, Moscow, 1983.

Received December 26, 2018; revised April 29, 2019; accepted May 5, 2019.

\section{Author information}

Dovlet M. Dovletov, Department of Mathematics, Near East University, Nicosia, TRNC, Mersin 10, Turkey.

E-mail: dovlet.dovletov@gmail.com 